\title{
Desde Brasil: vanguardia en antropología
}

\author{
Elena Azaola
}

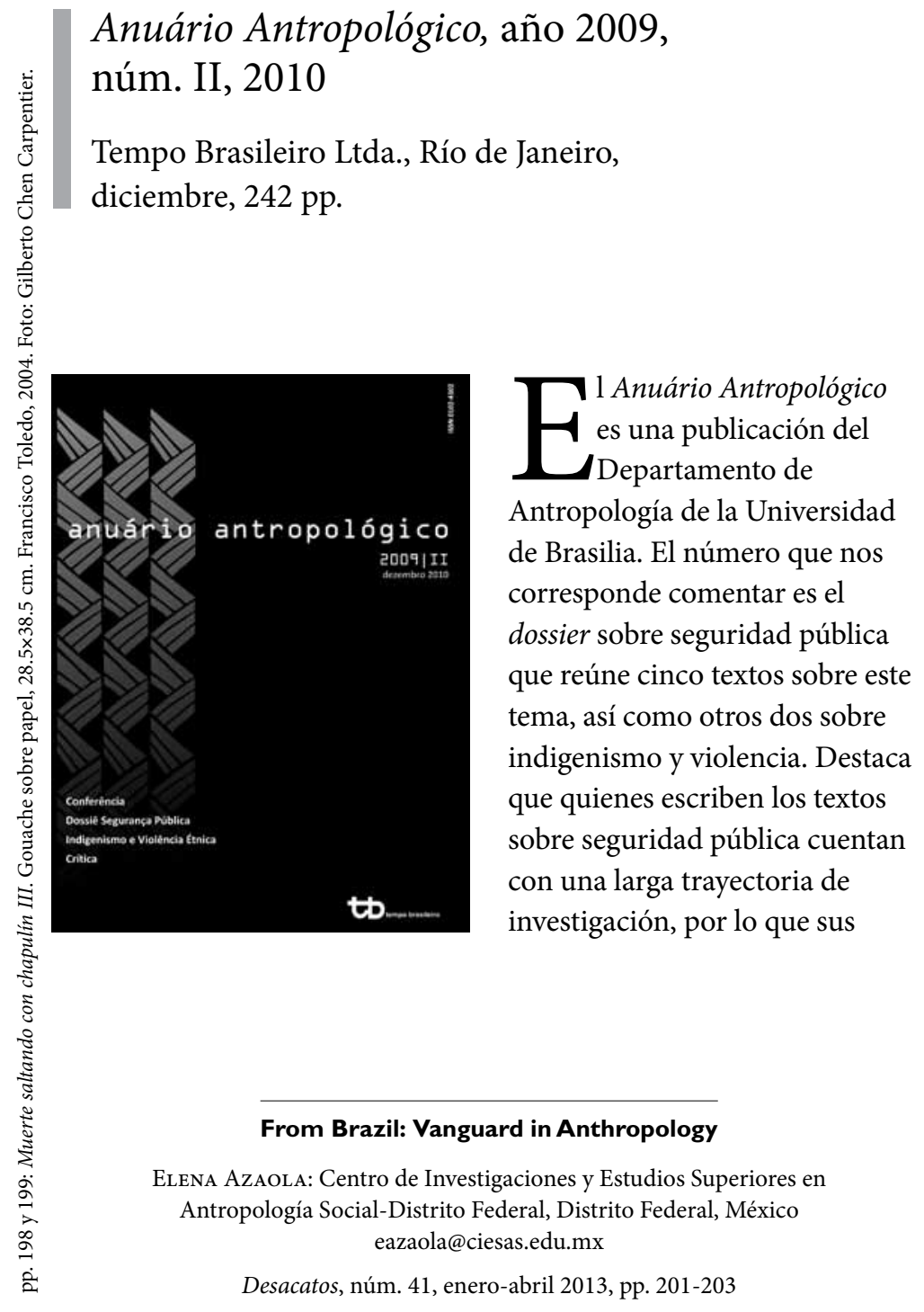

reflexiones son el resultado de un valioso conocimiento décadas en las que se han empeñado en mirar un mismo objeto desde ángulos diversos. El volumen inicia con una conferencia que Otávio Velho impartió en la VIII Reunión de Antropología del Mercosur, la cual tuvo por objeto honrar la memoria de Roberto Cardoso de Oliveira. El autor destaca el papel que Cardoso de Oliveira desempeñó al ocuparse del pensamiento crítico en Latinoamérica y al haber desarrollado las nociones de interculturalidad, procesos de hibridación, fricción interétnica, así como por la manera en que lidió con lo que llamó el predominio de un 
discurso eurocéntrico, al que solía aludir no sin cierto dejo de ironía. Velho corrige una de sus propias afirmaciones en el sentido de que Roberto Cardoso de Oliveira no habría creado una escuela de pensamiento, sino algo más sutil que tenía que ver con un modelo, un estilo, ya que señala que ahora piensa que es precisamente su modelo y su estilo los que le permitieron crear una especie de escuela "o tal vez simplemente de camaradería que se extiende a través del tiempo" (p. 18).

El primer texto sobre seguridad pública es del reconocido especialista Roberto Kant de Lima, quien se ocupa de lo que denomina distintas una perspectiva comparada. Tanto Kant de Lima como Cardoso de Oliveira han mostrado de manera más que convincente la pertinencia de hacer uso de las herramientas antropológicas para abordar el análisis comparativo de los sistemas jurídicos. En este caso, Kant de Lima emplea el concepto de sensibilidad legal propuesto por Geertz para explorar, desde una perspectiva comparativa, las bases culturales del derecho. Sus reflexiones derivan de haber efectuado durante más de 20 años dichos análisis comparados de los sistemas jurídicos, primero de Estados Unidos y Brasil, y más tarde de Argentina, Canadá y Francia. Compara, de este modo,

sistemas de justicia donde existe una amplia participación de los ciudadanos, como en los jurados estadounidenses, con aquellos otros que confían sólo en los veredictos que emiten los jueces calificados. Su intención es develar de qué manera aparecen en éste y en otros rasgos las diferentes "sensibilidades jurídicas". Describe al brasileño como un sistema mixto que tiene rasgos propios tanto del modelo de justicia inquisitorial como del acusatorio, pero en el que hay un claro predominio de los

funcionarios del Estado por encima de los ciudadanos, en contraste con lo que ocurre en los sistemas acusatorios en los que existe una mayor igualdad entre las partes. No obstante que el modelo brasileño integra reglas que son propias de sistemas que se encuentran basados en principios opuestos, en el fondo, según el especialista, termina reflejando la desigualdad, que es uno de los rasgos característicos de su estructura social.

El segundo artículo, de José Maria Nóbrega y Jorge Zeverucha, analiza la violencia homicida en el Nordeste brasileño y a través de un minucioso análisis de datos refuta la tesis que encuentra en la pobreza y la desigualdad la explicación de la violencia. En el caso que estudian, explican que tal relación causal no se confirma ya que mientras la pobreza y la desigualdad se han reducido, la violencia se ha incrementado, lo que atribuyen tanto a que la mejoría económica ha incrementado los niveles de consumo de alcohol y drogas como a la ineficiencia y corrupción de las instituciones encargadas de investigar y sancionar los homicidios. Un tercer texto, de Michel Misse, estudia los intercambios o negociaciones que tienen lugar a partir de la persecución y la sanción de los delitos donde siempre se opera con márgenes de discrecionalidad en los que se origina el intercambio de bienes o mercancías políticas ilícitas. Deja en claro que no existe una moral universal, sino que en cada contexto local hay balances distintos entre lo legal/ilegal, lo formal/informal, de donde se generan bienes o mercancías políticas con las cuales los funcionarios pueden negociar, si bien casi siempre en perjuicio de la moral pública más que de la privada.

El cuarto texto, de Jacqueline Sinhoretto, sitúa su análisis en el dilema planteado por Roberto DaMatta y Roberto Kant de Lima entre prácticas sociales jerárquicas y representaciones jurídicas igualitarias, lo que da lugar a lógicas jurídicas contradictorias cuyos agentes son a menudo acusados por abusos, cuando se trata de abusos comunes y tolerados en otras esferas de la práctica social. Se inscribe en la corriente de 
trabajos como los que han realizado Luis Roberto Cardoso de Oliveira y Michel Misse, quienes cuestionan la noción de sociedad disciplinaria propuesta por Foucault en contextos como el brasileño, en el que coexisten lógicas jurídicas igualitarias que conviven con prácticas jerárquicas que ponen en duda la posibilidad de que los individuos internalicen reglas dado que no sabrían a cuál sistema de reglas atenerse, particularmente en sus interacciones con agentes del Estado. Muestra también la aplicación fructífera del concepto de campo de Bourdieu para analizar el campo de la justicia en Brasil, con sus agentes y prácticas, formales e informales, visto como un campo de administración de conflictos. Se refiere entonces a las lógicas de administración de conflictos que corresponden a jerarquías de rituales, de personas y de tipos de conflictos. En cuanto a los conflictos de personas que tienen una alta jerarquía política, social o económica, la autora ilustra con citas de casos concretos de personas sometidas a la justicia con estas características. Se trata de un sistema que, en la manera de lidiar con los diferentes tipos de conflicto, deja claras las prioridades y, sobre todo, las desigualdades.

El quinto y último de los textos sobre seguridad pública es el de Ana Paula Mendes de Miranda, que se ocupa de cómo la reivindicación por la libertad religiosa entre la comunidad afrobrasileña tiene más que ver con la discriminación étnica de la que este grupo ha sido objeto y de cómo la reivindicación de la libertad religiosa es en realidad una reivindicación por los derechos en general de este grupo. En este sentido, el hecho de llevar los derechos por la libertad religiosa a la esfera pública ha sido una forma de mostrar la intolerancia religiosa como una forma más de discriminación. Completan la edición de este espléndido volumen, cuya lectura recomiendo ampliamente, dos artículos sobre situaciones de violencia en comunidades indígenas que no hacen sino confirmar lo dicho en los textos anteriores en cuanto a la desigualdad y la discriminación como dos de los rasgos característicos de la estructura social brasileña. Rasgos que, por cierto, no pueden dejar de expresarse en su sistema jurídico entendido como el campo o la arena donde se dirimen y se administran todo tipo de conflictos. 